\title{
O efeito da variabilidade do tempo de viagem no modal aéreo
}

\author{
Fernanda David Weber ${ }^{1}$, Luiz Afonso dos Santos Senna ${ }^{2}$, Ana Margarida Larrañaga Uriarte ${ }^{3}$, Carla \\ Schwengber ten Caten ${ }^{4 凶}$
}

1Universidade Federal de Pelotas, fernanda.weber@ufpel.edu.br

2Universidade Federal do Rio Grande do Sul, isenna@producao.ufrgs.br

3Universidade Federal do Rio Grande do Sul, analarrau@gmail.com

4Universidade Federal do Rio Grande do Sul, carlacaten@gmail.com

\section{Recebido:}

20 de dezembro de 2016

Aceito para publicação:

27 de setembro de 2017

Publicado:

29 de outubro de 2017

Editor de área:

Anderson Ribeiro Correia

Palavras-chaves:

Variabilidade do tempo de viagem,

Preferência declarada,

Transporte aéreo.

\section{Keywords:}

Travel time variability,

Stated preference,

Air transportation.

DOI:10.14295/transportes.v25i3.1295

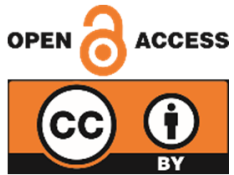

\begin{abstract}
RESUMO
A análise da variabilidade do tempo de viagem está ganhando interesse entre os pesquisadores e planejadores. Este trabalho avalia o comportamento dos usuários do transporte aéreo diante da presença da variabilidade de tempo de viagem. Especificamente, determina o comportamento dos usuários do transporte aéreo em relação a esta variabilidade, propensos, neutros ou avessos; identifica e caracteriza esta variabilidade na escolha do voo e compara o impacto das diferentes características envolvidas. Dados de preferência declarada foram coletados e modelos de escolha discreta estimados para modelar o comportamento dos usuários. Os resultados obtidos mostraram que os usuários do transporte aéreo são avessos à variabilidade do tempo de viagem. A comparação entre os impactos das variáveis mostrou que valorizam principalmente o custo da passagem na escolha do voo, seguido da existência de uma escala no deslocamento entre origem e destino, e por último o tempo e variabilidade do tempo de viagem.
\end{abstract}

\begin{abstract}
The analysis of travel time variability is gaining interest among researchers and planners. This work evaluates the behavior of air transport users in the presence of variability of travel time. Specifically, it determines the behavior of air transport users in relation to this variability, prone, neutral or averse; Identifies and characterizes this variability in flight choice and compares the impact of the different characteristics involved. Stated preference data were collected and discrete choice models estimated to model user behavior. The results showed that the users of the air transport are averse to the variability of travel time. The comparison between the impacts of the variables showed that they mainly value the cost of the passage in the choice of flight, followed by the existence of a scale in the displacement between origin and destination, and finally the time and variability of the travel time.
\end{abstract}

\section{INTRODUCTION}

Na sociedade moderna, as atividades econômicas, culturais e sociais são determinadas pelo horário do relógio. Até mesmo o fluxo de tráfego é regulado pelo relógio. 0 comportamento da maioria dos indivíduos reflete essa regulamentação. Interações com os outros, especialmente em casa e no trabalho, exigem que uma determinada parcela do padrão de comportamento de qualquer pessoa deva ser temporalmente e espacialmente fixo e recorrente, ou seja, repetitivo (Huff e Hanson, 1986).

0 tempo de viagem não é uma constante, e sim uma variável. Diversos componentes do sistema de tráfego interferem direta e indiretamente na variabilidade deste tempo. Assim, é possível afirmar que o conceito de variabilidade do tempo de viagem parte do princípio de que as escolhas das pessoas, em relação ao trajeto a ser realizado durante a viagem, devem ser tomadas sem o real conhecimento do tempo de viagem envolvido. Logo, não serão capazes de prever o tempo de viagem ou o horário de chegada ao destino sem antes iniciar a viagem (Li et al., 2010). 
O conhecimento dos usuários dos sistemas de transporte a respeito da probabilidade de ocorrência de um determinado tempo de viagem está relacionado com as diferentes fontes de variabilidade no tempo de viagem. Todos os modos de transporte podem ser mais ou menos confiáveis, mas sempre há um grau de irregularidade envolvida em qualquer viagem. A relevância da irregularidade ou da variabilidade do tempo de viagem para cada viajante depende, entre outros aspectos, da restrição do horário de chegada e do motivo da viagem.

Assim como nas várias dimensões e modos de transporte, o transporte aéreo também sofre com atrasos. Esse modal desempenha um papel central para as atividades econômicas. No caso brasileiro, em virtude de suas dimensões continentais, este tipo de transporte assume importância ainda mais significativa, tanto em relação ao desenvolvimento econômico quanto às atividades de lazer e culturais (Weber et al., 2012).

0 crescimento econômico e de renda, somados à precariedade de outros modais de transporte levaram a uma expansão da aviação comercial no Brasil. Nos anos 1990, teve início a política de flexibilização da aviação comercial brasileira, que removeu, de forma gradual, o controle sobre as variáveis econômicas do setor. 0 novo modelo de gestão, unido ao aumento do poder aquisitivo da população gerado pela estabilidade econômica do país, vem impulsionando fortemente o mercado aéreo. Muitas pessoas retomaram suas viagens utilizando este modal, e outras fizeram suas primeiras viagens de avião (Weber et al., 2012).

Em contrapartida, os avanços tecnológicos das aeronaves, cada vez maiores e mais pesadas, trouxeram problemas na infraestrutura aeroportuária (Rodolpho, 1997). 0 que se observa então, é que a demanda aumentou significativamente na primeira década do século XXI, mas a infraestrutura dos aeroportos não conseguiu acompanhar tal crescimento do mercado. Esse fator pode gerar atrasos nas viagens do modal aéreo.

Apesar do sistema de transporte aéreo contar com uma infraestrutura exclusiva, sem a interação com outros modais, garantindo-lhe um tráfego mais regulado e distribuído de forma mais eficiente (Fosgerau et al., 2008), atraso nos voos tem sido uma situação frequente nos aeroportos brasileiros, independentemente da companhia aérea. Qualquer atraso, mesmo que pequeno, no início da cadeia de deslocamentos pode levar a consequências prejudiciais aos viajantes, independentemente do modal utilizado (Rietveld et al., 2001). Por exemplo, um atraso no horário de abertura das portas da aeronave para iniciar o embarque pode levar a uma decolagem posterior ao horário marcado. Esse fato incorre em alterações no serviço adquirido; ou seja, o viajante não chegará ao aeroporto de destino no horário programado. Caso o atraso seja significativo, o viajante pode acabar por perder o voo de conexão, ou até mesmo perder o compromisso ou oportunidade de negócio, razão pela qual realizou a viagem.

Portanto, entender os efeitos da variabilidade do tempo de viagem no comportamento dos usuários do transporte aéreo pode se tornar uma vantagem competitiva para as empresas aéreas. Essa variável pode influenciar não somente a decisão com relação ao horário de partida, mas também a escolha da companhia aérea.

Diversos estudos que analisam a variabilidade do tempo de viagem e o comportamento dos usuários em estudos de transporte rodoviário e ferroviário foram reportados na literatura (Small et al., 1999; Bates et al., 2001; Brownstone e Small, 2005; Bhat e Sadesai, 2006; Hollander, 2006; Asensio e Matas, 2008; Hensher et al., 2011; Durán-Hormazábal e Tirachini, 2016; Engelson e Fosgerau, 2016; Charlotte et al., 2017). Entretanto, poucos estudos abordam este tema para o transporte aéreo. Os trabalhos desenvolvidos para o transporte aéreo se referem principalmente ao transporte de carga (por exemplo Andersson et al., 2016; Zhang et al., 2017), não sendo encontrados estudos que analisem o comportamento dos usuários frente à variabilidade do tempo de viagem. Este trabalho preenche essa lacuna e avalia o comportamento dos usuários do transporte aéreo diante da presença da variabilidade de tempo de viagem. Especificamente, este trabalho apresenta três objetivos: (i) determinar o comportamento dos usuários do transporte aéreo em relação a esta variabilidade, caracterizando se são propensos, neutros ou avessos; (ii) identificar se a variabilidade do tempo de viagem influencia a escolha do voo e como esta 
varia com as características dos indivíduos; e (iii) comparar o impacto das características da viagem envolvidas na escolha de um voo. Os resultados obtidos, assim como o modelo do comportamento dos usuários estimado neste estudo, poderão ser utilizados em estudos futuros para quantificar os custos de tempo de viagem e variabilidade do tempo de viagem associados à implantação de projetos de transportes.

Este artigo está organizado da seguinte forma: a seção 2 trata da variabilidade do tempo de viagem em estudos de transportes; a seção 3 apresenta a modelagem da variabilidade do tempo de viagem em transportes; na seção 4, os procedimentos metodológicos do estudo; e na seção 5 é realizada a análise dos dados e a estimação do modelo. Na seção 6 estão dispostas as conclusões do estudo.

\section{A VARIABILIDADE DO TEMPO DE VIAGEM EM TRANSPORTES}

O conceito de variabilidade do tempo de viagem é fundamental e está subentendido em qualquer análise de demanda de viagem. Origina-se, primeiramente, das necessidades e desejos das pessoas em participar de várias atividades que estão espacialmente distantes. Essas necessidades e desejos são determinados pelas características de cada indivíduo, que incluem ciclo de vida, estilo de vida e diversos fatores socioeconômicos (Pas, 1987).

Os modelos de demanda de viagem visam explicar as diferenças no comportamento de viagem das pessoas em termos das suas características sociodemográficas e da localização de suas residências. Quando se estuda comportamento em transportes (travel behaviour), há um consenso de que um sistema de transportes sem congestionamento não é um objetivo factível, realista (Banister, 2008). Congestionamentos e outras fontes de variabilidade nos tempos de viagem necessariamente conduzem à necessidade de questionamentos sobre o que seriam níveis aceitáveis ou razoáveis de congestionamentos, tendo como consequência o fato de que apenas a minimização do tempo de viagem possa não ser mais a premissa básica do comportamento. Desta forma, estudar a valoração apenas do tempo médio de viagem pode representar uma proposição excessivamente simplista. Sendo assim, se faz necessária a análise do comportamento frente a uma distribuição de tempos de viagem (Alves, 2014).

Segundo Alves (2014), ao se considerar uma distribuição de tempos de viagem, verifica-se a importância de se estudar questões relacionadas à confiabilidade dos sistemas de transporte. Diversos estudos mostram que a confiabilidade, em especial, a dos tempos de viagem, é uma característica altamente desejável para um sistema de transporte. Em muitos casos, os usuários valorizam mais um ganho na confiabilidade do tempo de viagem (tempo médio) do que uma redução no tempo médio de viagem. (Bates et al., 2001; Liu et al., 2004; Small et al., 1999).

Segundo Bates et al. (2001), a confiabilidade está associada ao conceito estatístico da variabilidade (consistência de um conjunto de medições). A confiabilidade do tempo de viagem pode representar a distribuição dos tempos de viagem por um período de tempo. A partir desta distribuição pode ser calculado o tempo médio de viagem (média da distribuição) e a variabilidade do tempo de viagem (desvio padrão). Batley (2007) e Fosgerau et al. (2008) discutiram a diferença entre os termos confiabilidade e variabilidade, mas ambos ainda são utilizados. Resumidamente, pode-se dizer que a confiabilidade dos tempos de viagem é o reflexo da variabilidade dos mesmos junto aos usuários do sistema (Alves, 2014).

\section{MODELANDO A VARIABILIDADE DO TEMPO DE VIAGEM EM TRANSPORTES}

Diversas idealizações, princípios e modelos, baseados em teorias particulares foram formulados para prever e/ou analisar a formação de preferências e o comportamento de escolha em diferentes contextos de aplicação (Timmermans, 2010). Os modelos de escolha discreta (Ben-Akiva e Lerman, 1985), que se baseiam no princípio da racionalidade são os mais utilizados em transportes, e com maior frequência quando se trata de estimar o valor da variabilidade do tempo de viagem. Geralmente, os modelos são estimados com dados coletados a partir de experimentos de preferência declarada (PD), ou de escolhas reveladas (PR) utilizando distribuições objetivas de tempo de viagem (Carrion e Levinson, 2012; Li et al., 2010). Na Figura 1 são apresentados os modelos que utilizam a abordagem da função utilidade na 
modelagem de transportes.

Dentre as abordagens apresentadas na Figura 1, optou-se por utilizar a abordagem da utilidade esperada apresentada por Senna (1994a; 1994b). A teoria da utilidade esperada é baseada na tomada de decisão sob risco. A escolha do modelo baseou-se na viabilidade de se obter modelos lineares ou nãolineares para as variáveis envolvidas no estudo. Além disso, o modelo de Senna alia a teoria microeconômica na sua concepção, permitindo que seja possível identificar o comportamento do público-alvo frente ao risco. Neste estudo, o risco é representado pela variabilidade do tempo de viagem.

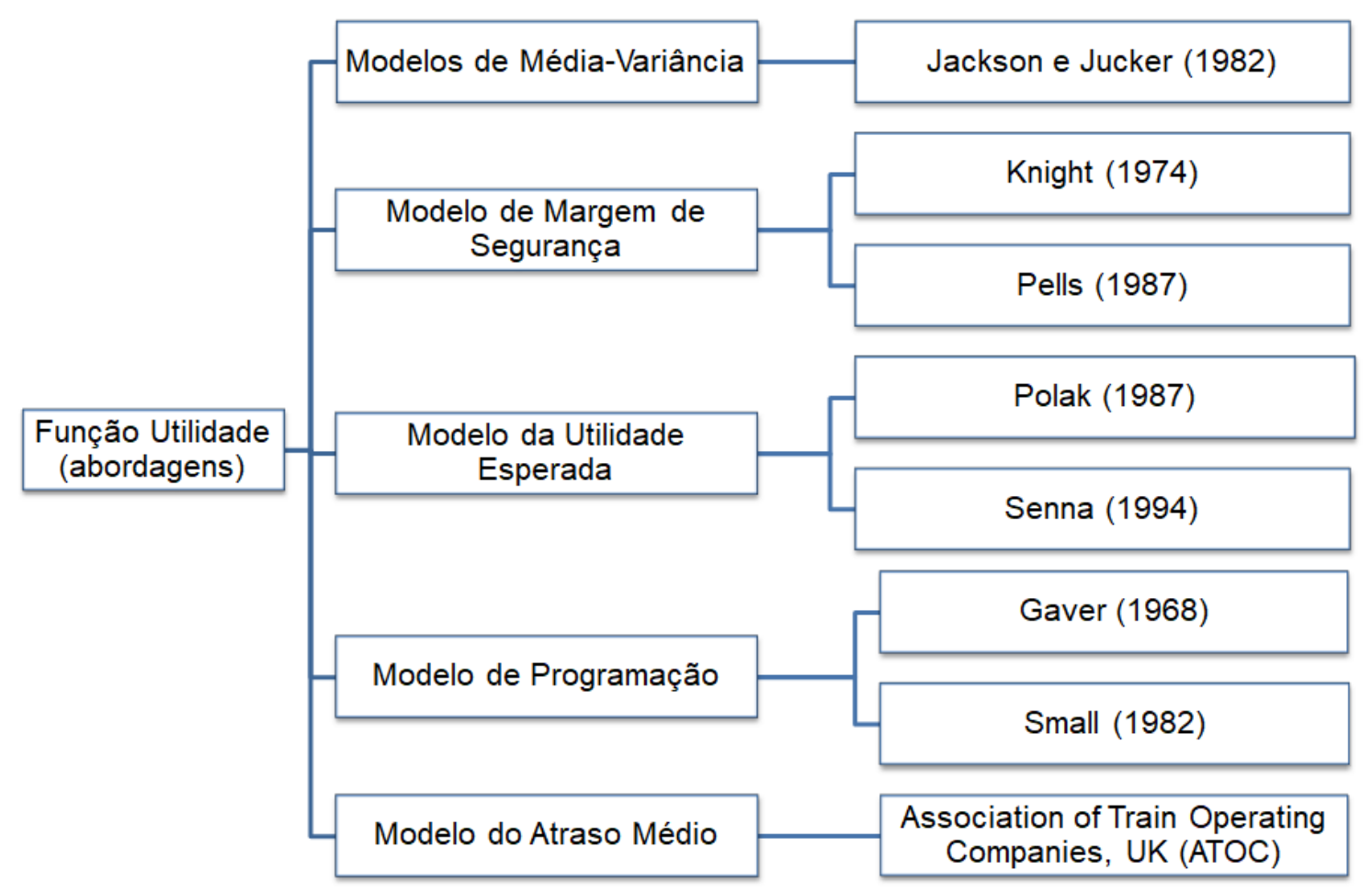

Figura 1. Abordagens da função utilidade em transporte para a variabilidade do tempo de viagem Fonte: Adaptado pelos autores (Carrion e Levinson, 2012; Li et al., 2010; Senna, 1994a)

\subsection{Modelo da utilidade esperada de Senna}

O modelo de Senna trata de situações de comportamento sob risco, e a forma funcional do modelo é que determina as atitudes dos tomadores de decisão nestas situações. Tais formas funcionais podem ser definidas com base em análises de regressão de experimentos (i.e., situações de trade-off, trocas compensatórias, que fornecem observações para estimar a função utilidade) ou formas computacionalmente convenientes (Hazell e Norton, 1986). O conceito de utilidade é oriundo da teoria do consumidor, representa a satisfação ou benefício que um indivíduo percebe pelo consumo de bens ou serviços. Senna (1994a) propôs uma forma generalizada para representar à utilidade, baseada no modelo proposto por Polak (1987), onde esta é dada por um termo algébrico de grau $\beta$ e um parâmetro $\gamma_{1}$,como mostra a Equação 1. Em estudos de transportes, geralmente, as funções de utilidade apresentam sinal negativo, indicando o desejo dos indivíduos de minimizar o tempo (e custo) da viagem, utilizando o termo (des)utilidade para representar esta condição.

$$
U=\gamma_{1} * t^{\beta}
$$

Neste caso, a Equação (1) pode ser escrita em termos da utilidade esperada, também através da aplicação do operador da expectativa. 0 resultado é um polinômio de grau $\beta$, apresentado na Equação (2). 


$$
E(U)=\gamma_{1} *\left\{\left[E\left(t^{\frac{\beta}{2}}\right)\right]^{2}+\operatorname{Var}\left(t^{\frac{\beta}{2}}\right)\right\}
$$

Nota-se que, na formulação apresentada por Senna, os termos que envolvem a média e a variância não são vistos separadamente, como é o caso da formulação proposta por Polak (1987). Além disso, a formulação de Senna apresenta certas propriedades. 0 parâmetro $\beta$ mensura o grau de aversão/propensão ao risco dos viajantes, baseado em teorias microeconômicas. Outro ponto a ser considerado é que o valor do tempo e o valor da variabilidade dependem diretamente da distribuição dos tempos de viagem.

\subsection{Comportamento dos indivíduos em relação ao risco}

Um dos primeiros estudos sobre tomada de decisão em situações de risco pode ser atribuída à Bernoulli (1738, traduzido por Sommer, 1954), que buscou explicar o motivo das pessoas possuírem antipatia ao risco, e analisar a variação desta antipatia com o aumento da renda. Após esse estudo, surgiu o interesse da incorporação do risco no processo de tomada de decisão e no comportamento de escolha em ciências aplicadas. No caso de transportes, esta abordagem pode ser considerada relativamente recente (Timmermans, 2010).

O comportamento dos indivíduos pode ser classificado em três grupos: a) propensão ao risco (i.e., aceita riscos); b) neutralidade ao risco (indiferentes ao risco) e c) aversão ao risco (i.e., não gostam de situações de risco). Estes conceitos são parte de uma teoria bem conhecida em economia, análise de utilidade esperada, que é qualificada por Friedman e Savage (1948) como uma "extensão bem simples da análise ortodoxa da utilidade" (Senna, 1994a). A Figura 2 apresenta uma visão geral da função utilidade esperada para comportamentos de aversão ao risco, propensão ao risco e neutralidade ao risco. Considerando que em estudos de transportes, a função de utilidade apresenta sinal negativo, foi representada no gráfico a (des)utilidade (valores opostos aos de utilidade).

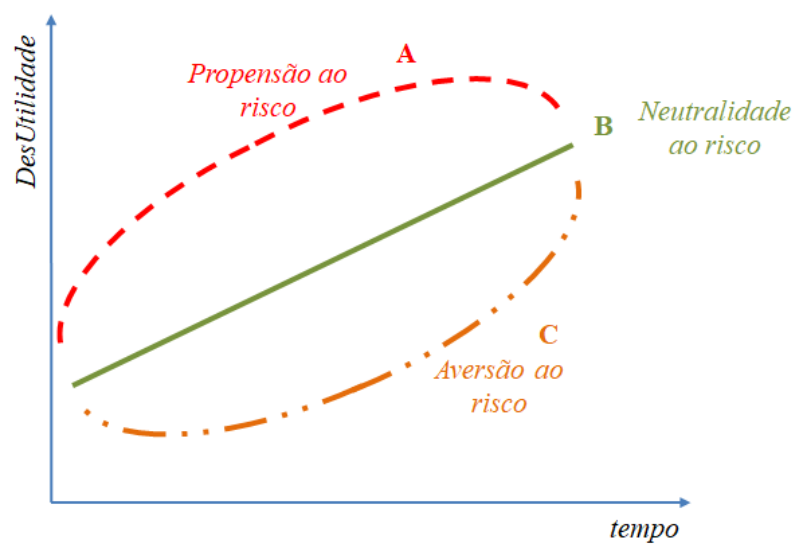

Figura 2. Função utilidade para diferentes comportamentos frente ao risco Fonte: Adaptado pelos autores (Senna, 1994a)

A condição de neutralidade indica que o indivíduo está interessado apenas no valor do tempo esperado e é totalmente alheio ao risco. Ao se determinar uma função de utilidade geral, como a apresentada na Equação (1), verifica-se que esta condição é válida para o valor $\beta$ igual a 1. Para um indivíduo com comportamento de aversão ao risco indica que não está interessado em variabilidade do tempo de viagem. Ao contrário, um indivíduo com este comportamento pode estar disposto a pagar prêmios elevados para garantir que não haverá atrasos, caso as penalidades por estes sejam altas. Partindo-se da função de utilidade geral, Equação (1) esta condição é satisfeita para valores de $\beta>1$. Já o comportamento de 
um indivíduo que seja propenso ao risco indica que o mesmo prefere um resultado incerto a um determinado resultado com o mesmo valor esperado. A função utilidade que identifica esta situação é estritamente côncava, onde a propensão ao risco pode ser identificada para valores de $\beta<1$.

\section{PROCEDIMENTOS METODOLÓGICOS}

A avaliação do comportamento dos usuários do transporte aéreo foi realizada através de modelos econométricos, utilizando a abordagem da função utilidade esperada proposta por Senna (1994a). 0 desenvolvimento deste trabalho foi realizado a partir de quatro etapas. A primeira envolveu a seleção dos atributos que foram utilizados no procedimento de modelagem. Já na segunda etapa, um projeto experimental foi elaborado utilizando um desenho fatorial completo, permitindo que todos os efeitos e suas combinações pudessem ser mensurados. A terceira etapa contemplou a coleta dos dados, envolvendo desde planejamento da pesquisa até a efetiva realização da mesma. A quarta e última etapa envolveu a estimativa de modelos de escolha discreta e a identificação do comportamento dos usuários do modal aéreo.

\subsection{Seleção dos atributos}

A seleção dos atributos que foram utilizados no processo de modelagem foi baseada na revisão da literatura e em uma pesquisa qualitativa desenvolvida para este estudo. Os atributos selecionados no estudo foram: (i) Tempo de viagem, (ii) Variabilidade do tempo de viagem, (iii) Custo e (iv) Escala.

A revisão de estudos sobre o comportamento dos usuários em transporte permitiu identificar que a avaliação do comportamento dos indivíduos frente ao risco, se refere à avaliação do comportamento frente à variabilidade do tempo de viagem. Esta variabilidade é geralmente apresentada ao público-alvo na forma de uma distribuição de tempos de viagem, abordagem adotada no presente estudo. Além disso, o custo da passagem envolvido para os deslocamentos e suas possíveis variações também foram considerados (Custo).

Não foram encontrados na literatura muitos estudos envolvendo a variabilidade do tempo de viagem no modal aéreo. Assim, com o intuito de averiguar a existência de algum atributo específico ao modal aéreo, foi realizada uma pesquisa qualitativa junto ao público-alvo, através de entrevistas individuais. A pesquisa qualitativa resultou na inclusão do atributo Escala, que também pode ser associado ao atributo transbordo, quando o estudo é no modal rodoviário ou ferroviário.

\subsection{Projeto experimental}

Definidos os atributos, foram determinados os níveis para cada atributo. Tempo de viagem, Variabilidade do tempo de viagem e Custo foram considerados com três níveis (alto, médio e baixo), para garantir a análise de uma possível relação não linear. Escala foi considerada com dois níveis (com ou sem escala, i.e., dummy). Os valores adotados para os níveis dos atributos foram estipulados com base na realidade do mercado aéreo. Por se tratar de um fatorial completo, foram consideradas todas as possíveis combinações entre os níveis de todos os atributos estudados, o que garantiu não só a análise dos efeitos principais, mas também suas interações.

Os valores para o custo foram determinados a partir do grau de planejamento de um viajante, o que pode garantir tarifas mais acessíveis quando a compra ocorre com maior tempo de antecedência. 0 nível alto considerou a compra do bilhete três dias antes da data da viagem. 0 nível médio considerou a compra com uma antecedência de quinze dias, enquanto que o nível baixo considerou a antecedência de um mês.

Os valores considerados para os atributos tempo de viagem e variabilidade do tempo de viagem foram os mesmos. 0 nível baixo considerou que o tempo médio de viagem (média da distribuição de tempos de viagem apresentada) fosse de quinze minutos a mais do que o tempo regular do trecho avaliado no estudo. 0 nível médio considerou uma adição de trinta minutos ao tempo regular médio, enquanto 
que o nível alto considerou um acréscimo de sessenta minutos ao tempo regular médio, sendo tais incrementos o resultado da média da distribuição do tempo de viagem regular. 0 tempo médio regular foi definido considerando a média dos tempos de viagem apresentados pelas companhias aéreas para o trecho estudado. 0 mesmo foi realizado na definição do tempo médio regular com a presença da escala. Já os valores dos níveis da variabilidade do tempo de viagem foram definidos como os mesmos do tempo de viagem: baixo, quinze minutos; médio, trinta minutos; e alto, uma hora. Porém, como a variabilidade do tempo de viagem foi apresentada na forma de distribuição de tempos de viagens, foram consideradas distribuições de tempos de viagem que resultaram em desvios padrão nos valores dos níveis.

\subsection{Coleta dos dados}

Os dados foram coletados através da técnica de preferência declarada, uma das mais utilizadas na modelagem da variabilidade do tempo de viagem (Bates et al., 2001; Batley e Ibáñez, 2009; Fosgerau et al., 2008; Hensher, 2001; Li et al., 2010; Noland et al., 1998; Small et al., 1999, 2005). Essa técnica garante que se obtenham mais dados com uma amostra menor devido à obtenção de múltiplas respostas de cada entrevistado. A técnica de preferência declarada trabalha com a apresentação de diferentes cenários aos entrevistados, coletando seus comportamentos frente a uma série de situações hipotéticas apresentadas, e não somente obtendo dados do comportamento já realizado pelos indivíduos (preferência revelada). Por essa razão, permite trabalhar com tamanhos de amostra menores e analisar o comportamento frente a situações não existentes na realidade.

0 projeto de experimento resultou em um estudo envolvendo 54 possíveis situações de escolha (03 x 03 x 03 x 02), que foram blocados em seis conjuntos (blocos) contendo nove cenários cada (Ortúzar e Willumsen, 2011). A escolha da escala semântica utilizada baseou-se na Transformada de Berkson-Theil, que determina a associação entre a escala semântica e a numérica. Esta pode ser vista na Tabela 1.

Tabela 1: Associação entre escalas semântica e numérica, segundo transformada de Berkson-Theil

\begin{tabular}{ll}
\hline Escala Semântica de Escolha & Probabilidades \\
\hline Certamente a alternativa A & 0,9 ou $90 \%$ \\
Provavelmente a alternativa A & 0,7 ou $70 \%$ \\
Indiferente & 0,5 ou $50 \%$ \\
Provavelmente a alternativa B & 0,3 ou $30 \%$ \\
Certamente a alternativa B & 0,1 ou $10 \%$ \\
\hline Fonte: Adaptado de Ortúzar e Willumsen (2011)
\end{tabular}

\subsection{Estimativa do modelo}

O modelo foi estimado segundo o método dos mínimos quadrados, aplicado ao modelo Logit Binário na sua forma linearizada. 0 Logit Binário permite a aplicação da transformada de Berkson-Theil para mensurar a probabilidade de escolha de um cenário frente a outro. A estimativa do modelo seguiu a abordagem da utilidade esperada proposta por Senna, que permite a identificação do comportamento do público-alvo do estudo frente ao risco, baseado nos conceitos econométricos. Ou seja, de forma mais robusta. Neste processo, foram considerados os efeitos principais dos atributos avaliados, assim como suas interações. A inclusão das variáveis socioeconômicas e suas interações com os atributos principais também foi contemplada durante a estimativa do modelo.

\section{ANÁLISE DOS RESULTADOS}

Esta seção apresenta a caracterização dos dados coletados e resultados da estimação dos modelos.

\subsection{Caracterização da amostra}

A coleta de dados foi realizada em outubro de 2014, tendo duração de 20 dias, no saguão do terminal de passageiros, nas áreas de check-in e áreas de espera e alimentação do Aeroporto Internacional Salgado 
Filho. Foram entrevistados passageiros em todos os turnos, selecionados de forma aleatória. Desse modo, buscou-se abranger todos os tipos de viajantes: trabalho e lazer, principalmente. Mesmo supondose que muitos viajantes a trabalho prefiram viajar nas primeiras horas do dia, e retornem no final do mesmo, foi possível encontrar muitos passageiros viajando por ambos os motivos ao longo do dia e da semana.

Tabela 2: Caracterização da amostra

\begin{tabular}{|c|c|c|c|c|c|}
\hline & & Cat. & Classe & & \\
\hline & Sexo & 1 & Homem & 275 & $46 \%$ \\
\hline & & 2 & Mulher & 321 & $54 \%$ \\
\hline & Estado Civil & 1 & Casado & 282 & $47 \%$ \\
\hline & & 2 & Solteiro & 242 & $41 \%$ \\
\hline & & 3 & Separado & 54 & $9 \%$ \\
\hline & & 4 & Viúvo & 51 & $4 \%$ \\
\hline & Grau de Instrução & 1 & Ensino Fundamental & 1 & $0 \%$ \\
\hline & & 2 & Ensino Médio & 149 & $25 \%$ \\
\hline & & 3 & Ensino Superior & 319 & $54 \%$ \\
\hline & & 4 & Pós-Graduação & 127 & $21 \%$ \\
\hline & Residência & 1 & Porto Alegre & 182 & $31 \%$ \\
\hline & & 2 & RMPA* & 100 & $17 \%$ \\
\hline & & 3 & Interior do RS & 118 & $20 \%$ \\
\hline Caracteristicas & & 4 & Outro Estado ou País & 196 & $33 \%$ \\
\hline & Faixa Etária & 1 & Até 25 anos & 112 & $19 \%$ \\
\hline & & 2 & De 26 a 35 anos & 166 & $28 \%$ \\
\hline & & 3 & De 36 a 45 anos & 104 & $17 \%$ \\
\hline & & 4 & De 46 a 55 anos & 93 & $16 \%$ \\
\hline & & 5 & De 56 a 65 anos & 72 & $12 \%$ \\
\hline & & 6 & Mais de 65 anos & 49 & $8 \%$ \\
\hline & Renda Familiar (mensal) & 1 & Até $\mathrm{R} \$ 1.400,00$ & 8 & $1 \%$ \\
\hline & & 2 & $\mathrm{R} \$ 1.400,01$ a $\mathrm{R} \$ 2.800,00$ & 27 & $5 \%$ \\
\hline & & 3 & $\mathrm{R} \$ 2.800,01$ a $\mathrm{R} \$ 4.900,00$ & 96 & $16 \%$ \\
\hline & & 4 & $\mathrm{R} \$ 4.900,01$ a $\mathrm{R} \$ 7.000,00$ & 178 & $30 \%$ \\
\hline & & 5 & $\mathrm{R} \$ 7.000,01$ a $\mathrm{R} \$ 10.500,00$ & 144 & $24 \%$ \\
\hline & & 6 & $\mathrm{R} \$ 10.500,01$ a $\mathrm{R} \$ 14.000,00$ & 67 & $11 \%$ \\
\hline & & 7 & Mais de $R \$ 14.000,00$ & 76 & $13 \%$ \\
\hline & Motivo da Viagem & 1 & Trabalho ou Negócios & 185 & $31 \%$ \\
\hline & & 2 & Lazer ou Turismo & 357 & $60 \%$ \\
\hline & & 3 & Outros & 54 & $9 \%$ \\
\hline & Frequência de Viagem & 1 & De 02 ou mais vezes ao mês & 77 & $13 \%$ \\
\hline & (no modal aéreo) & 2 & De 02 ou mais vezes ao semestre & 129 & $22 \%$ \\
\hline & & 3 & De 02 ou mais vezes ao ano & 199 & $33 \%$ \\
\hline & & 4 & Outras & 191 & $32 \%$ \\
\hline & Destino das Viagens & 1 & Sempre o mesmo & 42 & $7 \%$ \\
\hline Característicge da Viagem & & 2 & Frequentemente o mesmo & 132 & $22 \%$ \\
\hline Caracteristicas da Viagem & & 3 & Variados & 422 & $71 \%$ \\
\hline & Pagamento da Passagem & 1 & Próprio & 372 & $62 \%$ \\
\hline & & 2 & Empregador ou Cliente & 110 & $18 \%$ \\
\hline & & 3 & $3^{\circ}$ Pessoa & 114 & $19 \%$ \\
\hline & Importância da Pontualidade & 1 & Ambos, embarque e desembarque & 515 & $86 \%$ \\
\hline & & 2 & Embarque & 62 & $10 \%$ \\
\hline & & 3 & Desembarque & 15 & $3 \%$ \\
\hline & & 4 & Indiferente & 4 & $1 \%$ \\
\hline & & 5 & Não é importante & 0 & $0 \%$ \\
\hline
\end{tabular}

Foram entrevistados 697 usuários do transporte aéreo. As respostas foram validadas, comprovando que as escolhas ocorreram conforme o esperado, implicando na identificação de indivíduos lexicográficos (escolhem sempre a alternativa na qual a combinação dos atributos avaliados é melhor) e indivíduos cativos de uma alternativa. Dentre os indivíduos lexicográficos, 63 escolheram somente com base no menor custo (9\%); e 38 somente pela regularidade (6\%). 
Esses indivíduos foram retirados da análise, pois os modelos de escolha discreta buscam identificar os pontos de trade-offs entre as variáveis estudadas, o que não ocorreu nestes casos. 0 banco de dados final contou com 596 entrevistas, totalizando 5.364 respostas, representando 85\% do banco de dados inicial. A Tabela 2 apresenta as características socioeconômicas e de viagem da amostra pesquisada.

A distribuição da amostra por gênero coincide com a distribuição da população do último censo obtida para a cidade de Porto Alegre (IBGE, 2010), mostrando a representatividade da amostra em relação ao gênero dos indivíduos. Aproximadamente 50\% dos entrevistados residem na cidade de Porto Alegre e na região metropolitana. Os usuários entrevistados possuem alto nível de instrução, $75 \%$ possuem curso superior e pós-graduação e maior renda familiar do que a média da cidade (IBGE, 2010). Aproximadamente $50 \%$ dos entrevistados apresentam renda familiar mensal maior que $\mathrm{R} \$ 7.000$. A maioria das viagens realizadas pelos respondentes são viagens a lazer, com destinos variados, e não muito frequentes (65\% dos respondentes viajam menos que 02 vezes por semestre). A maioria dos entrevistados reportou a importância da pontualidade, tanto no embarque quanto no desembarque.

\subsection{Estimação dos modelos}

Os modelos estimados segundo a abordagem de Senna (1994a; 1994b) não assume uma forma funcional pré-determinada. Esta é dada em virtude do valor de $\beta$, conforme foi apresentada na Equação (2). A adaptação desta equação para o presente estudo é apresentada na Equação (2). Assim como no modelo geral proposto por Senna (1994a; 1994b), o termo $\left\{\left[\mathrm{E}\left(\mathrm{t}^{\beta / 2}\right)\right]^{2}+\left[\sigma\left(\mathrm{t}^{\beta / 2}\right)\right]^{2}\right\}$ envolve a média e a variabilidade do tempo de viagem (Tempo de viagem, Variabilidade do tempo de viagem). Entretanto, estes termos não são vistos separadamente, como é o caso de outras formulações presentes na literatura (Jackson e Jucker, 1982; Polak, 1987), obtendo como resultado um único parâmetro para representar ambos. A seguir, as variáveis Tempo de viagem, Variabilidade do tempo de viagem serão representadas pela expressão combinada de ambas, expressas no termo $\left\{\left[\mathrm{E}\left(\mathrm{t}^{\beta / 2}\right)\right]^{2}+\left[\sigma\left(\mathrm{t}^{\beta / 2}\right)\right]^{2}\right\}$.

$$
E(U)=\varphi+\alpha *\left\{\left[E\left(t^{\frac{\beta}{2}}\right)\right]^{2}+\left[\sigma\left(t^{\frac{\beta}{2}}\right)\right]^{2}\right\}+\delta * \text { Custo }+\lambda * \text { Escala }
$$

A primeira etapa da construção do modelo foi definir qual valor de $\beta$ retorna o melhor modelo $\left(\mathrm{R}_{\mathrm{a}}{ }^{2}\right)$. 0 projeto de experimento permitiu que o valor $\beta$ variasse de zero a dois, $0<\beta \leq 2$. Portanto, foram testados diversos valores de $\beta$ dentro deste intervalo, e a relação destes com o $\mathrm{R}_{\mathrm{a}}^{2}$ são apresentados na Figura 3. 0 melhor modelo resultou do valor de $\beta$ igual a 1,30, onde o $\mathrm{R}_{\mathrm{a}}{ }^{2}$ foi de 0,4575 (a confirmação ocorreu ao comparar a quinta casa decimal). A opção em utilizar mais de duas casas decimais para este fator foi justamente viabilizar a exata relação do comportamento frente ao risco. 0 valor resultante, $\beta=$ 1,30, indica que os usuários do transporte aéreo têm aversão ao risco.

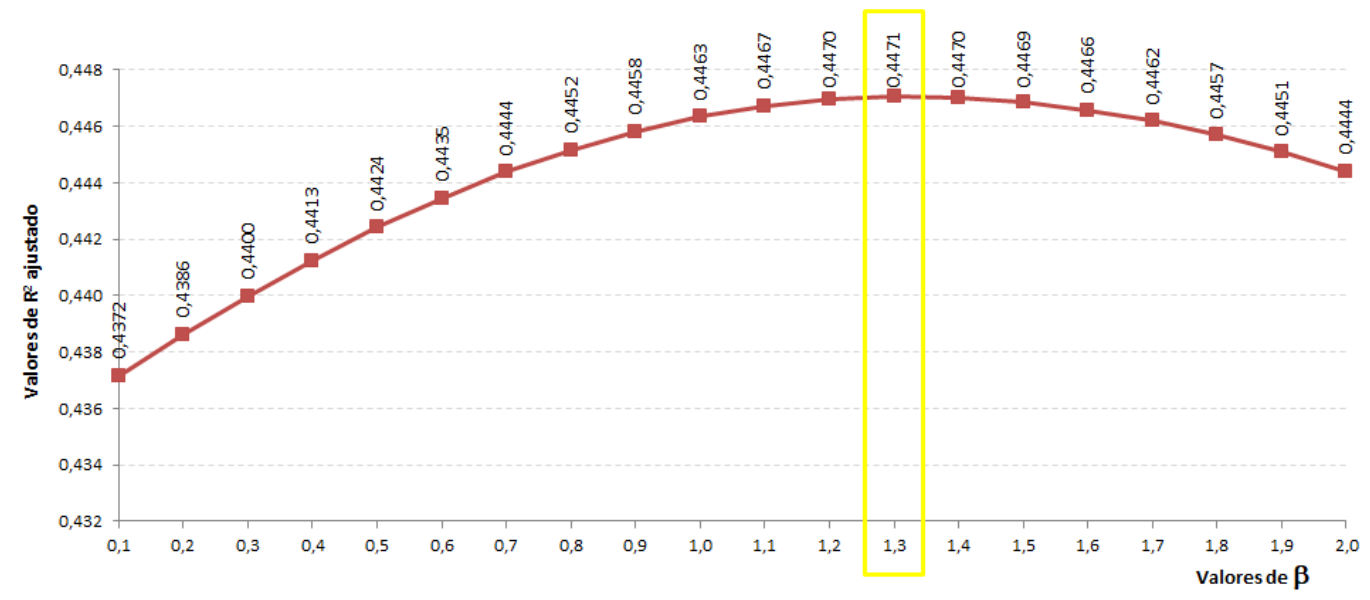

Figura 3. Relação entre os valores de $\beta$ e o $\mathrm{Ra}^{2}$ para o modelo de Senna 
Definido o valor $\beta(1,30)$ partiu-se para a estimação do modelo inserindo os efeitos principais e algumas interações, além dos fatores socioeconômicos. A Tabela 3 apresenta os parâmetros e as variáveis contempladas no modelo final estimado. As variáveis apresentadas na Tabela 2, que representam as características dos indivíduos, também foram incorporadas na estimação dos modelos.

Tabela 3: Modelos da Utilidade Esperada contendo efeitos secundários e variáveis socioeconômicas

\begin{tabular}{|c|c|c|}
\hline \multirow{2}{*}{ Variáveis } & \multicolumn{2}{|c|}{ Modelo SENNA $(\beta=1,30)$} \\
\hline & Parâmetros & Valor-p \\
\hline Intercepto & 1,144 & 0,000 \\
\hline Escala (0/não - $1 /$ sim) & 0,1159 & 0,001 \\
\hline Custo (R\$) & $-0,01234$ & 0,057 \\
\hline$\left\{[\mathrm{E}(\mathrm{t} / 2)]^{2}+[\sigma(\mathrm{t} / 2)]^{2}\right\} \quad(\min )$ & $-0,344$ & 0,000 \\
\hline Renda Familiar & 0,2104 & 0,000 \\
\hline Frequência de viagem & $-0,0686$ & 0,078 \\
\hline Custo $^{2}$ & 0,000012 & 0,000 \\
\hline$\left\{\left[\mathrm{E}\left(\mathrm{t}^{/ 2}\right)\right]^{2}+[\sigma(\mathrm{t} / 2)]^{2}\right\}^{*}$ Faixa Etária & $-0,0809$ & 0,000 \\
\hline$\left\{[\mathrm{E}(\mathrm{t} / 2)]^{2}+[\sigma(\mathrm{t} / 2)]^{2}\right\}^{*}$ Renda Familiar & $-0,1604$ & 0,000 \\
\hline$\left\{[\mathrm{E}(\mathrm{t} / 2)]^{2}+[\sigma(\mathrm{t} / 2)]^{2}\right\}^{*}$ Frequência de viagem & $-0,0737$ & 0,021 \\
\hline$\left\{[\mathrm{E}(\mathrm{t} / 2)]^{2}+[\sigma(\mathrm{t} / 2)]^{2}\right\}^{*}$ Motivo de viagem & 0,1205 & 0,013 \\
\hline$\{[\mathrm{E}(\mathrm{t} / 2)]+[\sigma(\mathrm{t} / 2)]\}^{2}$ & $-1,465$ & 0,001 \\
\hline Renda Familiar*Motivo de viagem & $-0,1148$ & 0,000 \\
\hline Motivo de viagem*Pagamento da passagem & $0,0151^{*}$ & 0,141 \\
\hline $\mathrm{R}_{\mathrm{a}}^{2}$ & \multicolumn{2}{|c|}{0,5224} \\
\hline Total de respondentes & \multicolumn{2}{|c|}{522} \\
\hline Total de observações & \multicolumn{2}{|c|}{4.697} \\
\hline
\end{tabular}

$\left(^{*}\right)$ Significativo para nível de confiança superior a $80 \%$

A Tabela 3 mostra que os parâmetros estimados resultaram nos sinais esperados. Os coeficientes relacionados ao custo (Custo), tempo de viagem e variabilidade do tempo de viagem $\left(\left[\mathrm{E}\left(\mathrm{t}^{\beta / 2}\right)\right]^{2}+\left[\sigma\left(\mathrm{t}^{\beta / 2}\right)\right]^{2}\right)$ apresentaram sinal negativo, mostrando o desejo dos usuários de minimizar tempo, variabilidade do tempo e custo de viagem. Com relação aos efeitos secundários, verificou-se que aqueles que envolvem a variável tempo de viagem associada à variabilidade do tempo de viagem $\left[\mathrm{E}\left(\mathrm{t}^{\beta / 2}\right)\right]^{2}+\left[\sigma\left(\mathrm{t}^{\beta / 2}\right)\right]^{2}$, também possuem o sinal esperado. As interações desta variável com Faixa Etária, Renda Familiar, Frequência de viagem indicam que os indivíduos de maior renda, faixa etária mais alta e passageiros mais frequentes são mais sensíveis ao tempo e variabilidade do tempo de viagem. A interação desta variável com Motivo de viagem apresentou sinal positivo, mostrando que os passageiros que viajam a trabalho são mais sensíveis ao tempo e variabilidade do tempo de viagem.

Frequência de viagem apresentou sinal negativo. Acredita-se que a razão de tal resultado seja oriunda do conhecimento adquirido pelo respondente com o acúmulo de dados a respeito de viagens em um mesmo modal. Também foram estimados modelos inserindo as variáveis socioeconômicas: gênero, faixa etária e motivo de viagem; porém, nenhuma se mostrou significativa.

Ao verificar a interação entre os fatores socioeconômicos e de viagem, constatou-se que há relação entre Renda Familiar e Motivo de viagem. Esse resultado é esperado, uma vez que, quanto maior a renda, maior é o valor dispensado para lazer, viabilizando mais deslocamentos, sejam no modal aéreo, sejam nos demais. 0 mesmo é esperado em relação ao motivo trabalho da viagem. Pessoas com melhores salários geralmente ocupam cargos mais estratégicos nas empresas e mercado, o que leva novamente a mais deslocamentos.

A interação entre Motivo de viagem e Pagamento da passagem foi significativa, mostrando que passageiros que viajam a trabalho e pagam sua própria passagem são mais sensíveis à escolha do vôo e apresentam menor utilidade do que passageiros que viajam a lazer e/ou aqueles que viajam a trabalho, mas a passagem é paga por terceiros. Este resultado é esperado, verificando novamente o desejo dos usuários de minimizar os custos de viagens, especialmente nos deslocamentos a trabalho.

Os parâmetros estimados foram comparados relativizando os valores em relação a sua média (naive 
approach), de forma de analisar o impacto de cada um na escolha de um vôo. A comparação permitiu verificar que o impacto do Custo (3.6) é significativamente maior que o impacto do tempo e variabilidade (0.03), representado pelo termo $\left[\mathrm{E}\left(\mathrm{t}^{\beta / 2}\right)\right]^{2}+\left[\sigma\left(\mathrm{t}^{\beta / 2}\right)\right]^{2}$, e que a Escala $(0.06)$.

\section{CONCLUSÕES}

A análise da variabilidade do tempo de viagem está ganhando interesse entre os pesquisadores e planejadores. 0 congestionamento e a falta de confiabilidade dos sistemas de transporte tornam-se ocorrências diárias e uma grande preocupação para os prestadores de serviços e formuladores de política. No transporte aéreo, os impactos podem ser mais significativos que em outros modos, uma vez que os custos envolvidos nos deslocamentos costumam ser altos. 0 estudo realizado avaliou o comportamento dos usuários do modal aéreo diante da presença da variabilidade do tempo de viagem. A atitude dos usuários frente a esta variabilidade foi estudada para tráfego rodoviário e ferroviário, carecendo de estudos para o modal aéreo.

Modelos de escolha discreta forma utilizados para modelar o comportamento dos usuários do transporte aéreo. A função de utilidade utilizada foi proposta por Senna (1994a), a qual não limita a linearidade das variáveis envolvidas nos modelos e alia a teoria microeconômica na sua concepção, permitindo a identificação do comportamento do público-alvo frente ao risco. Os dados foram obtidos de uma pesquisa de preferência declarada, utilizando um projeto fatorial completo. A utilização de um projeto fatorial completo permitiu analisar com precisão possíveis interações entre as variáveis envolvidas.

Os resultados obtidos mostraram que o comportamento dos usuários não é linear e os usuários são avessos à variabilidade do tempo de viagem. 0 valor resultante do parâmetro $\beta$ (termo algébrico da função de utilidade) foi maior que um $(\beta>1)$, indicando que os viajantes do transporte aéreo possuem aversão ao risco, aqui representado pela variabilidade do tempo de viagem.

A análise dos parâmetros estimados mostra que os coeficientes relacionados ao custo (Custo), tempo de viagem e variabilidade do tempo de viagem $\left(\left[\mathrm{E}\left(\mathrm{t}^{\beta / 2}\right)\right]^{2}+\left[\sigma\left(\mathrm{t}^{\beta / 2}\right)\right]^{2}\right)$ apresentaram sinal negativo, mostrando o desejo dos usuários de minimizar tempo, variabilidade do tempo e custo de viagem. A interação entre os atributos modelados indicou que os indivíduos de maior renda, maior faixa etária e passageiros mais frequentes são mais sensíveis ao tempo e variabilidade do tempo de viagem. Adicionalmente, os passageiros que viajam a trabalho são mais sensíveis ao tempo e variabilidade do tempo de viagem.

A comparação entre os impactos das variáveis relativas à viagem permitiu verificar que os usuários pesquisados valorizam principalmente o custo da passagem para realizar uma viagem, seguido da existência de uma escala no deslocamento entre origem e destino, e por último o tempo e variabilidade do tempo de viagem.

A determinação do valor do tempo de viagem e da variabilidade do tempo de viagem não seguem as expressões tradicionais, devendo ser derivadas a partir das equações postuladas no estudo. Futuros estudos podem ser realizados neste sentido, de foram de quantificar o valor do tempo de viagem e da variabilidade do tempo de viagem para serem usados em estudos de transporte.

\section{REFERÊNCIAS}

Alves, B. B. (2014) O comportamento de viagens de acesso a aeroportos considerando a confiabilidade do tempo de viagem. Tese de Doutorado, Escola Politécnica, Universidade de São Paulo.

Andersson, M., M. Berglund, J. Flodén, C. Persson e J. Waidringer (2017) A method for measuring and valuing transport time variability in logistics and cost benefit analysis. Research in Transportation Economics, In Press, p.1-11. DOI:10.1016/j.retrec.2017.03.001

Asensio, J., e A. Matas (2008) Commuters' valuation of travel time variability. Transportation Research Part E: Logistics and Transportation Review, v.44, n.6, p. 1074-1085. DOI:10.1016/j.tre.2007.12.002

Banister, D. (2008) The sustainable mobility paradigm. Transport Policy, v.15, n. 2, p. 73-80. DOI:10.1016/j.tranpol.2007.10.005

Bates, J., J. Polak, P. Jones, e A. Cook (2001) The valuation of reliability for personal travel. Transportation Research Part E: Logistics and Transportation Review, v. 37, n. 2-3, p. 191-229. doi:10.1016/S1366-5545(00)00011-9 
Batley, R. (2007) Marginal valuations of travel time and scheduling, and the reliability premium. Transportation Research Part E: Logistics and Transportation Review, v. 43, n. 4, p. 387-408. DOI:10.1016/j.tre.2006.06.004

Batley, R., e J. N. Ibáñez (2009) Randomness in preferences, outcomes and tastes ; an application to journey time risk. International Choice Modelling Conference, p. 1-27. Harrogate, UK.

Ben-Akiva, M. e S. R. Lerman (1985) Discrete Choice Analysis: Theory and Application to Travel Demand. (s.l) MIT Press.

Bernoulli, D. (1954) Exposition of a new theory on the measurement of risk. Econometrica, v. 22, n. 1, p. 23-36. DOI: $10.2307 / 1909829$

Bhat, C., e R. Sadesai (2006) The impact of stop-making and travel time reliability on commute mode choice. Transportation Research Part B: Methodological, v. 40, n. 9, p. 709-730.

Brownstone, D. e K. A. Small (2005) Valuing time and reliability: assessing the evidence from road pricing demonstrations. Transportation Research Part A: Policy and Practice, v. 39, n. 4, p. 279-293. DOI:10.1016/j.tra.2004.11.001

Carrion, C. e D. Levinson (2012) Value of travel time reliability: A review of current evidence. Transportation Research Part A: Policy and Practice, v. 46, n. 4, p. 720-741. DOI:10.1016/j.tra.2012.01.003

Charlotte, C., L. M. Helene e B. Sandra (2017) Empirical estimation of the variability of travel time. Transportation Research Procedia, v. 25, p. 2769-2783. DOI:10.1016/j.trpro.2017.05.225

Durán-Hormazábal, E. e A. Tirachini (2016) Estimation of travel time variability for cars, buses, metro and door-to-door public transport trips in Santiago, Chile. Research in Transportation Economics, v. 59, p. 26-39. DOI:10.1016/j.retrec.2016.06.002

Engelson, L., e M. Fosgerau (2016) The cost of travel time variability: Three measures with properties. Transportation Research Part B: Methodological, v. 91, n. 555-564. D0I:10.1016/j.trb.2016.06.012

Fosgerau, M., K. Hjorth, C. Brems e D. Fukuda (2008) Travel time variability Definition and valuation Mogens Fosgerau. Report 1, DTU Transport.

Friedman, M. e L. J. Savage (1948) The Utility Analysis of Choices Involving Risk. The Journal of Political Economy, v. 56, n.4, p. 279-304. Obtido de http://www.jstor.org/stable/1826045

Hazell, P. B. R. e R. D. Norton (1986) Mathematical programming for economic analysis in agriculture. Macmillan Publishing Company, New York, USA.

Hensher, D. A. (2001) The valuation of commuter travel time savings for car drivers: evaluating alternative model specifications. Transportation, v. 28, p. 101-118.

Hensher, D. A., W. H. Greene e Z. Li (2011) Embedding risk attitude and decision weights in non-linear logit to accommodate time variability in the value of expected travel time savings. Transportation Research Part B: Methodological, v. 45, n. 7, p. 954-972. DOI:10.1016/j.trb.2011.05.023

Hollander, Y. (2006) Direct versus indirect models for the effects of unreliability. Transportation Research Part A: Policy and Practice, v. 40, n. 9, p. 699-711. DOI:10.1016/j.tra.2005.12.007

Huff, J., e S. Hanson (1986) Repetition and Variability in Urban Travel. Geographical Analysis, v. 18, n. 2, p. 97-114.

Jackson, W. B. e J. V. Jucker (1982) An empirical study of travel time variability and travel choie behaviour. Transportation Science, v. 16, n. 4, p. 460-475.

Li, Z., D. A. Hensher e J. M. Rose (2010) Willingness to pay for travel time reliability in passenger transport: A review and some new empirical evidence. Transportation Research Part E: Logistics and Transportation Review, v. 46, n. 3, p. 384-403. DOI:10.1016/j.tre.2009.12.005

Liu, H. X., W. Recker e A. Chen (2004) Uncovering the contribution of travel time reliability to dynamic route choice using realtime loop data. Transportation Research Part A: Policy and Practice, v. 38, n. 6, p. 435-453. DOI:10.1016/j.tra.2004.03.003

Noland, R. B., K. A. Small, P. M. Koskenoja e X. Chu (1998) Simulating travel reliability. Regional Science and Urban Economics, v. 28 , n. 5, p. 535-564.

Ortúzar, J. D. D. e L. G. Willumsen (2011) Modelling Transport (4th ed). Jonh Wiley \& Sons.

Pas, E. I. (1987) Intrapersonal Variability and Model Gooness-of-fit. Transportation Research Part A: Policy and Practice, v. 21A, n. 6, p. 431-438. DOI: 10.1016/0191-2607(87)900.

Polak, J. W. (1987) More General Model of individual departure time choice. PTRC Summer Annual Meeting (p. 247-258).

Rietveld, P., F. Bruinsma e D. van Vuuren (2001) Coping with unreliability in public transport chains: A case study for Netherlands. Transportation Research Part A: Policy and Practice, v. 35, n. 6, p. 539-559. DOI:10.1016/S09658564(00)00006-9

Rodolpho, R. (1997) Índices de dimensionamento de terminais de passageiros. Trabalho de Conclusão de Graduação, Instituto Tecnológico de Aeronáutica - ITA, Departamento de Ciência e Tecnologia Aeroespacial - DCTA.

Senna, L. A. D. S. (1994a) Users Response to Travel Time Variability. Doctor of Philosophy, Department of Civil Engineering, Institute of Leeds, The University of Leeds.

Senna, L. A. D. S. (1994b) The influence of travel time variability on the value of time. Transportation, v. 21, p. 203-228.

Small, K. A., R. B. Noland, X. Chu e D. Lewis (1999) Valuation of travel time savings and predictability in congested conditions for highway user cost estimation. NCHRP Report 431, Transportation Research Board, National Research Council.

Small, K. A., C. Winston e J. Yan (2005) Uncovering the distribution of motorists' preference for travel time and reliability. Econometrica, v. 73, n. 4, p. 1367-1382. Obtido de http://www.jstor.org/discover/10.2307/3598825?sid=21105516803263\&uid=3737664\&uid=2\&uid=4

Timmermans, H. (2010) On the (Ir)relevance of Prospect Theory in Modelling Uncertainty in Travel Decisions, European Journal of Transport and Infrastructure Research, v. 10, n. 4, p. 368-384. 
Weber, F. D., A. C. B. Mancuso, L. A. S. Senna e M. E. Echeveste (2012) Avaliação dos requisitos da qualidade em terminais aeroportuários: o caso do aeroporto Salgado Filho. XVII PANAM - Congreso Panamericano de Ingeniería de Tránsito, Transporte y Logística. Santiago, Chile.

Zhang, W., E. Jenelius e X. Ma (2017) Freight transport platoon coordination and departure time scheduling under travel time uncertainty. Transportation Research Part E: Logistics and Transportation Review, v. 98, p. 1-23. DOI:10.1016/j.tre.2016.11.008 